This item was submitted to Loughborough's Research Repository by the author.

Items in Figshare are protected by copyright, with all rights reserved, unless otherwise indicated.

\title{
Propagation of wedge acoustic waves along wedges embedded in water
}

PLEASE CITE THE PUBLISHED VERSION

http://dx.doi.org/10.1109/ULTSYM.1994.401763

PUBLISHER

(c) IEEE

VERSION

VoR (Version of Record)

LICENCE

CC BY-NC-ND 4.0

REPOSITORY RECORD

Krylov, Victor V.. 2012. "Propagation of Wedge Acoustic Waves Along Wedges Embedded in Water". figshare. https://hdl.handle.net/2134/9669. 
This item was submitted to Loughborough's Institutional Repository (https://dspace.lboro.ac.uk/) by the author and is made available under the following Creative Commons Licence conditions.

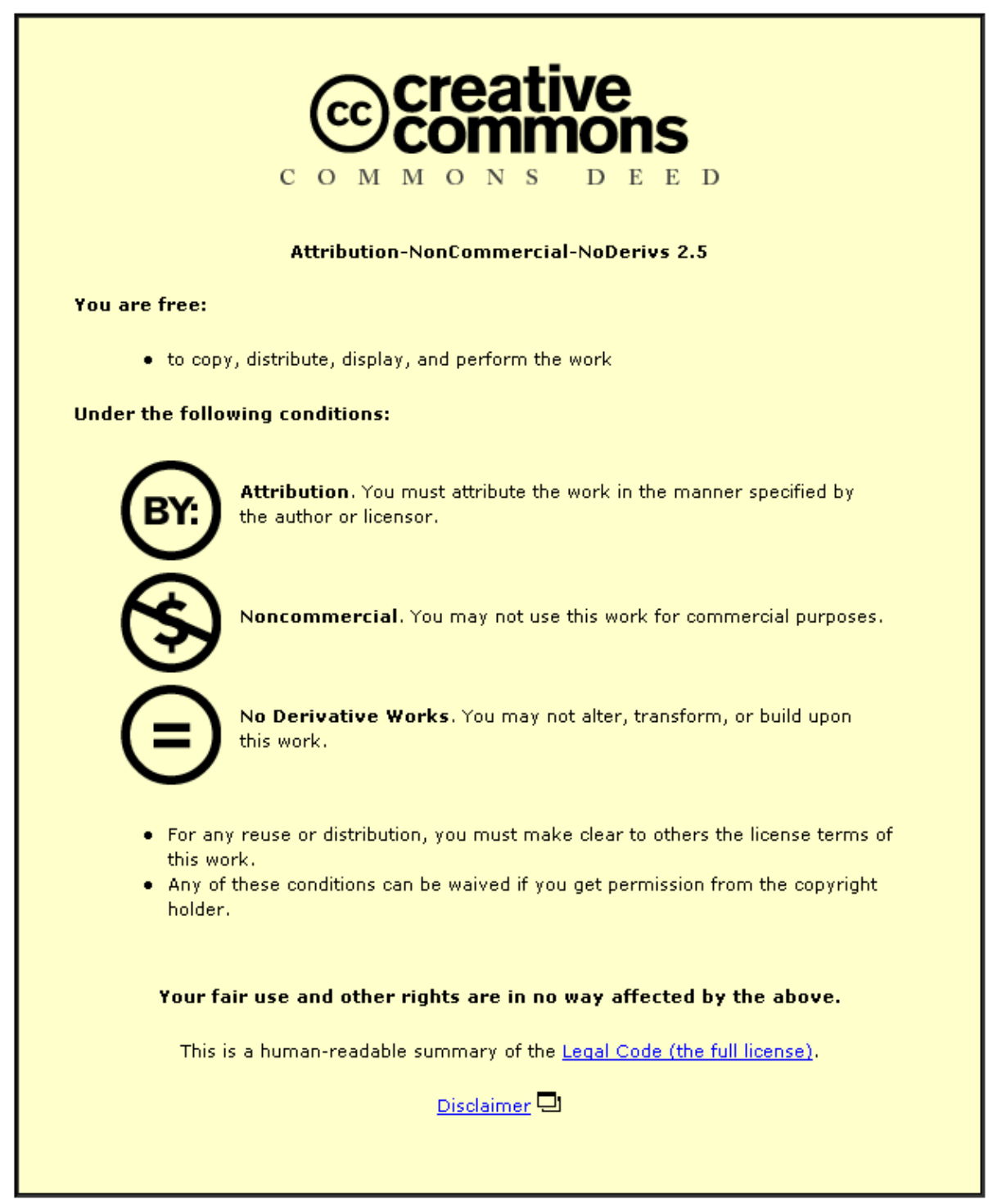

For the full text of this licence, please go to: http://creativecommons.org/licenses/by-nc-nd/2.5/ 


\title{
PROPAGATION OF WEDGE ACOUSTIC WAVES ALONG WEDGES IMBEDDED IN WATER
}

\author{
Victor V. Krylov \\ Centre for Research into the Built Environment, Nottingham Trent University, \\ Burton Street, Nottingham NG1 4BU, U.K.
}

\begin{abstract}
In this paper, the preliminary theoretical results are reported on the propagation of antisymmetric (lexural) wedge acoustic waves in solid wedges immersed in liquid. It is shown that in a subsonic regime of propagation, the influence of liquid may result in significant decrease of wedge wave velocities in comparison with their values in vacuum. For wedges made of such materials as rubber, the wedge-wave velocities may be as small as few meters per second. Wedge waves with such low propagation velocities may be used as a new source for propulsion of vessels in water similar to that used in nature by some fish, e.g. rays, which use wave-like motion of their wings for moving forward. The advantage of using wedge waves for wave-like propulsion of man-inhabited vessels in water is that the main body of a vessel would be virtually quiet because the wedge-wave vibration energy is concentrated near the wedge tips.
\end{abstract}

\section{INTRODUCTION}

Wedge acoustic waves are disturbances propagating along the tips of elastic wedges, their elastic energy being concentrated in the area of about one wave length near the wedge tips (Fig. 1).

These waves have been discovered in 1972 using numerical calculations $[1,2]$ and then investigated in a number of works with regard to their applications to nonlinear signal processing devices and to nondestructive testing of special engineering constructions (with edges) (see, e.g., [3-9]). However, almost all of these works considered propagation of wedge waves in contact with vacuum. Apart from the recent experimental work [7] no attention has been paid to understanding their interaction with liquids. Meanwhile such interaction may play an essential role in many practical phenomena, e.g., in the process of noise generation by propellers where a supersonic regime of wedge wave propagation should be important.

However, in our opinion, one of the most intriguing and promising practical application of wedge waves in liquids might be their use for in-water propulsion of ships and submarines which obviously should take place in the subsonic regime of propagation of wedge acoustic waves.

The principle of the proposed use of wedge acoustic waves for in-water propulsion is similar to that used in nature by fish of the ray family which apply a wave-like motion of their wings for moving forward. It is proposed to design special wing-like structures for supporting wedge elastic waves generated by vessel engines. These wedge waves will result in hydrodynamic forces applied from water to the wings to propel the vessel. Existing parts of the vessels, like keels of yachts and ships, could be used as the wing-like structures supporting antisymmetric wedge waves. In other cases, e.g. for small submarine vessels, special-purpose wings would be created (Fig.2).

The expected advantages of this new propulsion method over existing ones (e.g., using jets and propellers) are the following:

1. It is quiet, which is a particularly attractive feature for applications where minimal disturbance of wildlife is important;

2. It is probably more energy-efficient since it follow's nature (this, however, needs to be proved both theoretically and experimentally);

3. It is environmentally friendly (does not create cavitation) and relatively safe for people and wildlife;

4. It does not interfere with existing hydrodynamic properties of vessels when not in use (this is important for yachts with sails).

The unique benefit of using wedge waves (in comparison, e.g., with flexural waves in plates) for wave-like propulsion of man-inhabited vessels is that the main body of the vessel could be virtually quiet 
because the wedge wave-vibration energy is concentrated near the wedge tip.

To test the engineering feasibility of this new propulsion method, it is necessary to undertake detailed investigations of wedge acoustic wave propagation in contact with liquids, including the associated hydrodynamic phenomena. These should involve theoretical study of wedge wave excitation and propagation in wedge-like solid structures of different shapes and materials imbedded in water, investigation of hydrodynamic flows and motive forces generated by wedge waves, including the effect of large wave amplitudes. The above mentioned theoretical investigations should be supported by appropriate experimental studies. And finally, a working model of a vessel based on the wedge-wave effect should be designed, constructed and tested.

The present paper represents an initial part of this program, namely the theoretical demonstration of the existence of very slow antisymmetric (flexural) wedge waves in immersed solid wedges. This is necessary prerequisite of practical realisation of the proposed inwater propulsion method.

\section{EFFECT OF LIQUID ON PLATE WAVES}

In this section we derive the expression for the wavenumber of the lowest antisymmetric mode of a plate (flexural wave) imbedded in liquid. The results then will be used for the numerical calculations of the velocities of localised wedge modes using geometrical acoustics approach to the wedge wave description [4-6].

Despite the existing solutions based on thin plate theories (see, e.g., $[10,11]$ ), we rederive the expression for flexural wavenumber starting from the exact Rayleigh - Lamb equation for antisymmetric movement of a plate imbedded in liquid. This provides the possibility of future easy generalisation of the theory to thicker plates.

The equation we deal with has the form [12]

$$
\begin{aligned}
& \frac{\tanh \left(\frac{d}{2} \sqrt{k^{2}-k_{l}^{2}}\right)}{\tanh \left(\frac{d}{2} \sqrt{k^{2}-k_{t}^{2}}\right)}-\frac{4 k^{2} \sqrt{k^{2}-k_{l}^{2}} \sqrt{k^{2}-k_{t}^{2}}}{\left(2 k^{2}-k_{t}^{2}\right)^{2}} \\
& +i \frac{\rho_{f} k_{t}^{4} \sqrt{k^{2}-k_{l}^{2}}}{\rho_{s} \sqrt{k_{f}^{2}-k^{2}}\left(2 k^{2}-k_{t}^{2}\right)^{2} \tanh \left(\frac{d}{2} \sqrt{k^{2}-k_{t}^{2}}\right)}=0
\end{aligned}
$$

where $d$ is the plate thickness, $k=\omega / c$ is the wave number of propagating antisymmetric mode, $c$ is its phase velocity, and $\omega$ is the circular frequency, $k_{l}=\omega / c_{l}$ and $k_{t}=\omega / c_{t}$ are the wave numbers of longitudinal and shear acoustic waves in solids respectively, $c_{l}$ and $c_{t}$ are their velocities, $\rho_{s}$ and $\rho_{f}$ are respectively the mass densities of solid and liquid.

Substituting the expressions $k_{l}=\omega / c_{l}$ and $k_{t}=$ $\omega^{\prime} c_{t}$ into Eqn (1) and expanding the left hand side of (1) into power series of $d$ and $\omega$ (up to terms proportional to $\omega^{4}$ ), one can get the following dispersion equation for the lowest flexural mode of a plate imbedded in liquid:

$$
\begin{aligned}
& \frac{1}{2} \frac{\omega^{4}}{c_{t}^{4} k^{4}}-\frac{1}{6}\left(c_{l}^{2}-c_{t}^{2}\right) \frac{d^{2}}{c_{l}^{2} c_{t}^{2}} \omega^{2} \\
& +\frac{\rho_{f}}{\rho_{s}}-\frac{\omega^{4}}{c_{t}^{4} k^{4}} \frac{1}{d \sqrt{k^{2}-\omega^{2} / c_{f}^{2}}}=0
\end{aligned}
$$

Using the notation for flexural rigidity $D=\left(\rho_{s} c_{p}{ }^{2} / 12\right) d^{3}$, where $c_{p}=2 c_{t}\left(l-c_{t}^{2} / c_{l}^{2}\right)^{1 / 2}$ is the so called plate velocity, one can easily show that Eqn (2) coincides with the dispersion equation which is usually used in literature for flexural waves in thin plates faced to liquid at both sides. In the absence of liquid ( $\left.\rho_{f}=0\right)$ Eqn (2) reduces to the well known dispersion relation for a flexural wave in a thin plate.

For very slow plate modes $\left(k>>/ c_{f}\right)$ which are only considered in this paper, one can use the approximation of incompressible liquid, and Eqn. (2) may be simplified as

$$
\begin{aligned}
& \frac{1}{4} \frac{\omega^{4}}{c_{t}{ }^{4} k^{4}}-\frac{1}{12}\left(c_{l}^{2}-c_{t}^{2}\right) \frac{d^{2}}{c_{l}^{2} c_{t}^{2}} \omega^{2} \\
& +\frac{1}{2} \frac{\rho_{f}}{\rho_{s}} \frac{\omega^{4}}{c_{t}^{4} k^{4}} \frac{I}{k d}=0
\end{aligned}
$$

Solving Eqn (3) versus $\omega$ gives

$$
\omega=\frac{l}{\sqrt{3}} \frac{c_{t}}{c_{l}} k^{5 / 2} \sqrt{\rho_{s}} \frac{d^{3 / 2}}{\sqrt{\rho_{s} k d+2 \rho_{f}}} \sqrt{c_{l}^{2}-c_{t}^{2}}
$$

Taking into account that plates are thin ( $k d<<l)$ and assuming that $\rho_{f}$ is of the same order as $\rho_{s}$, i.e. considering plates imbedded in liquids rather than in gases, one can get the simplified expression

$$
\omega=\frac{1}{\sqrt{6}} k^{5 / 2} \frac{\sqrt{\rho_{s}}}{\sqrt{\rho_{f}}} d^{3 / 2} \frac{c_{t}}{c_{l}} \sqrt{c_{l}^{2}-c_{t}^{2}}
$$


which allows the explicit solution for the flexural wave number $k$ :

$$
k=\left[\sqrt{6} \frac{c_{l}}{c_{s}} \frac{1}{\sqrt{c_{l}^{2}-c_{t}^{2}}} \frac{\sqrt{\rho_{f}}}{\sqrt{\rho_{s}}} \frac{\omega}{d^{3 / 2}}\right]^{2 / 5}
$$

Eqn (6) will be used in further consideration as a starting point for the calculation of wedge acoustic wave velocities for wedges imbedded in liquid. Thus, all necessary information on the influence of liquid is contained in the expression for the wavenumber of flexural waves in immersed plates.

\section{GEOMETRICAL-ACOUSTICS THEORY}

In the framework of the geometrical acoustics approach to the description of wedge waves, a slender solid wedge is considered as a plate of a local variable thickness $d=x \Theta$, where $\Theta$ is the wedge angle and $x$ is the distance from the wedge tip measured in the middle plane (see Fig.1). The velocities of wedge acoustic modes are determined from the Bohr Sommerfeld relation [4-6]

$$
\int_{0}^{x_{t}}\left[k^{2}(x)-\beta^{2}\right]^{1 / 2} d x=m
$$

Here $\beta=\omega / c$ is the wavenumber of a wedge mode, $c$ is its phase velocity, $k(x)$ is a current local wavenumber of a flexural wave in a plate of variable thickness, $n=1,23, \ldots$ is the mode number, and $x_{t}$ is the so called ray turning point determined from the equation $k^{2}(x)-\beta^{2}=0$.

For example, in the case of a wedge in vacuum we have $k(x)=12^{1 / 4} k_{p}^{1 / 2}(\theta x)^{-1 / 2}, x_{t}=2 \sqrt{3} k_{p} / \Theta \beta^{2}$, where $k_{p}=$ $\omega^{\prime} c_{p}$, and the solution of Eqn (7) yields the simple analytical expression for wedge wave velocities

$$
c=c_{p} n \Theta / \sqrt{3}
$$

which agrees well with other theories and with the experimental results. Note that although, strictly speaking, the geometrical acoustics approach to the description of wedge waves is not valid for the lowest wedge mode $(n=1)$, in practice it provides quite accurate results for wedge wave velocities even in this case [4,5]. The analytical expressions for structure of wedge modes are rather cumbersome $[5,6]$ and are not displayed here.

To calculate the velocities of wedge waves in a wedge imbedded in liquid we will make use of the expression for $k(x)$ determined by Eqn (6). Substituting (6) into (7) and introducing the dimensionless notations $y=c / c_{t}, z=$ $d k_{t}=x \Theta \omega / c_{t}$ one can derive the following equation versus $y$ :

$$
\int_{0}^{(A y)}\left[\frac{A^{2}}{z^{6 / 5}}-\frac{1}{y^{2}}\right]^{1 / 2} d z=\pi n \theta
$$

where

$$
\begin{aligned}
& A=\sigma^{1 / 5}\left(\rho_{f} \rho_{s}\right)^{2 / 5}\left(1-c_{t}^{2} / c_{l}^{2}\right)^{-1 / 5} \\
& =\sigma^{1 / 5}\left(\rho_{f} \rho_{s}\right)^{2 / 5}[2(1-\sigma)]^{1 / 5}
\end{aligned}
$$

is a nondimensional parameter dependent on the relation of the mass densities of liquid and solid and on the Poisson ratio $\sigma$. Eqn (9) can not be solved analytically. The results of its numerical solution are discussed in the next section.

\section{NUMERICAL SOLUTION AND DISCUSSION}

The numerical solution of Eqn (9) has been carried out using standard Mathcad package for wedges made of aluminium $\left(\rho_{s}=2.7 \times 10^{3} \mathrm{~kg} / \mathrm{m}^{3}, c_{l}=6420 \mathrm{~m} / \mathrm{s}, \quad c_{t}=\right.$ $3110 \mathrm{~m} / \mathrm{s}, \sigma=0.35)$ and rubber $\left(\rho_{s}=0.93 \times 10^{3} \mathrm{~kg} / \mathrm{m}^{3}\right.$, $\left.c_{l}=1040 \mathrm{~m} / \mathrm{s}, \quad c_{t}=30 \mathrm{~m} / \mathrm{s}, \sigma=0.499\right)$. These yields $A=$ 1.014 - for aluminium and $A=1.474$ - for nubber. The calculated values of $y 1$ and $y 2$ corresponding to the two lowest order flexural wedge modes $(n=1,2)$ as functions of the wedge angle $\Theta$ for immersed aluminium and rubber wedges are shown on Figs. 3 and 4 respectively. The straight lines represent the corresponding solutions $Y 1$ and $Y 2$ for wedges in vacuum described by Eqn (8).

One can see that the influence of liquid results in noticeable decrease of wedge wave velocities in comparison with their values in vacuum. This decrease is a nonlinear function of a wedge apex angle $\theta$ and is more pronounced for small values of $\theta$. For example, for the lowest order wedge mode propagating in a sharp rubber wedge $(\theta=0.1 \mathrm{rad})$ imbedded in water, the wedge wave velocity is more than 5 times smaller than for a wedge in vacuum. This implies that for wedges made of rubberlike materials, the wave velocities may be as low as few meters per second. Wedge waves with such low propagation velocities might be suitable for use as a new source of in-water propulsion.

Note that according to the classical calculations of M. J. Lighthill for wave-like propulsion of a slender fish [13], the optimum value of velocity of the flexural motion of the fish body should be equal to $5 / 4$ of the desired swimming speed. In this case the swimming speeds attained by fish are "remarkably high in relation to their available muscle power". One can expect that similar optimum wedge wave velocity might exist also for a vessel propelled by wedge waves. Investigation of this problem is now in progress. 


\section{REFERENCES}

1. Lagasse, P.E., Electronics Letters, 8 (1972) 372-373.

2. Maradudin, A.A., Wallis, R.F., Mills, D.L. and Ballard, R.L., Phys. Rev. B6 (1972) 1106-1111.

3. McKenna, J., Boyd G.D. and Thurston, R.N., IEEE Trans. on Sonics and Ultrasonics, 21 (1974) 178-186.

4. Krylov, V.V., Proc. II Intermational Symp. on Surface Waves in Solids and Layered Structures, Vama, Bulgaria, 1989. World Scientific, Singapore (1990) 174. 189.

5. Krylov, V.V., Soviet Phys. - Tech. Phys. 35 (1990) $137-$ 140.

6. Krylov, V.V. and Parker, D.F., Wave Motion, 15 (1992) 185-200.

7. Chamuel, J.R., Proc IEEE Ultrasonics Symposium, Baltimore, MD, USA (1993) 313-318.

8. Jia, X., Auribault, D., De Billy, M. and Quentin, G., Proc IEEE Ultrasonics Symposium, Baltimore, MD, USA (1993) 637-640.

9. Krylov, V.V., Mayer, A.P. and Parker, D.F., Proc IEEE Ultrasonics Symposium, Baltimore, MD, USA (1993) 765-768.

10. Junger, M.C. and Feit, D. Sound, Structures and their Interaction. Publ. Acoust. Soc. Amer., New-York (1993).

11. Crighton, D.G., Dowling, A.P., Ffowcs Williams, J.E., Heck1, M. and Lepington, F.G., Modem Methods in Analytical Acoustics. Springer-Verlag, London, Berlin, Heidelberg et al. (1992).

12. Merkulov, L.G., Sov. Phys. Acoust., 10 (1964) 169-173.

13. Lighthill, M.J., J. Fluid Mech., 9 (1960) 305-317.

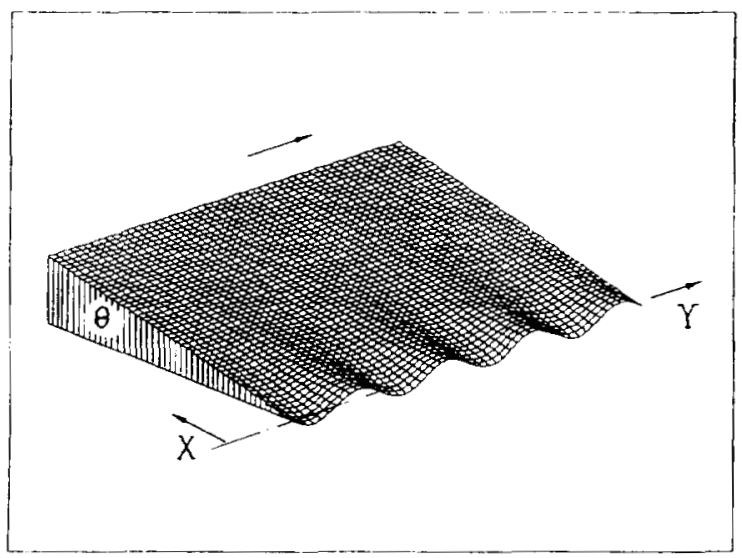

Fig.1 Antisymmetric wedge acoustic waves

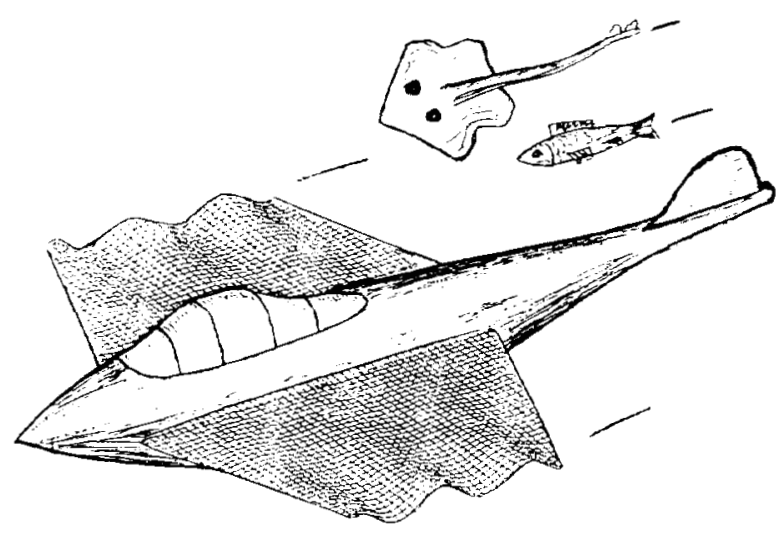

Fig.2 Using wedge waves for propulsion of a small submarine

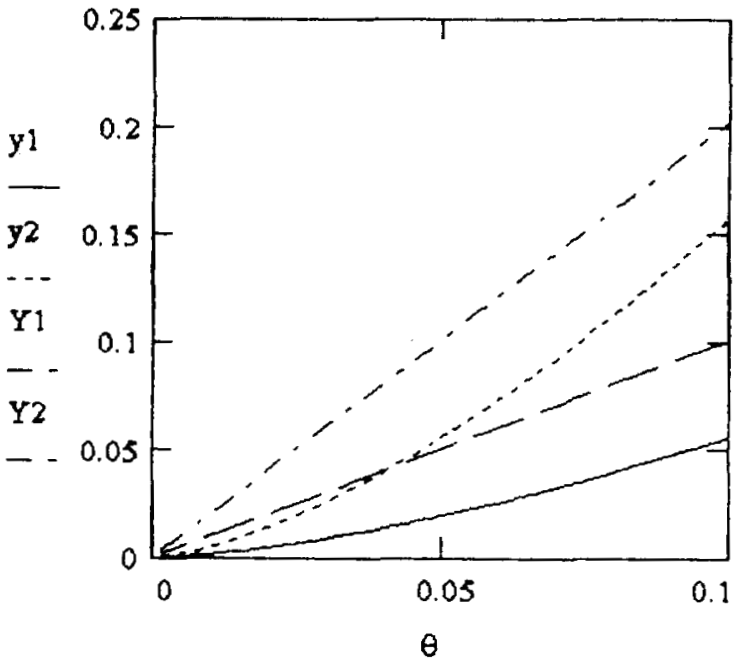

Fig.3 Normalised velocities of two wedge modes for aluminium wedges in water $(y 1, y 2)$ and in vacuum $(\mathbf{Y} 1, \mathbf{Y} 2)$

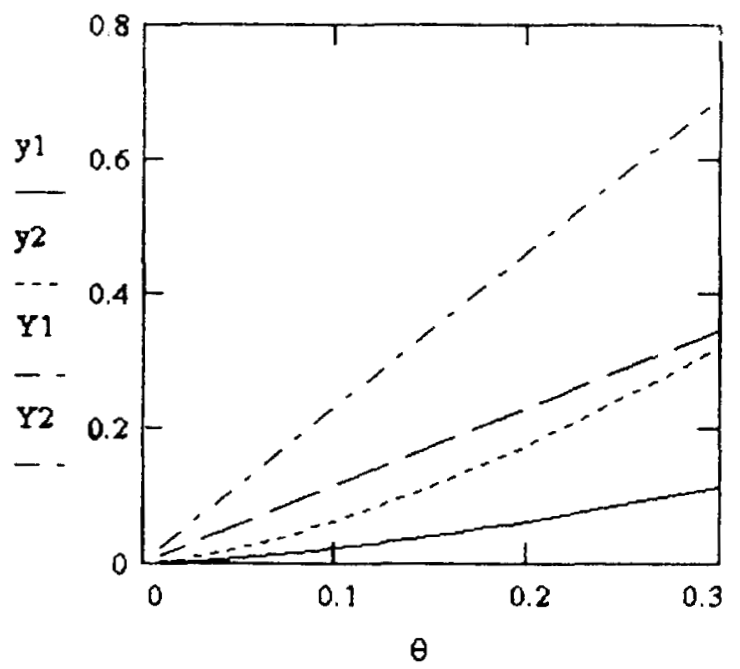

Fig.4 Normalised velocities of two wedge modes for rubber wedges in water $\left(y 1, y_{2}\right)$ and in vacuum (Y1,Y2) 